\title{
EDITORIAL
}

\section{La importancia de la investigación de enfermería en enfermedades crónicas}

A ctualmente el cáncer, la enfermedad vascular cerebral, la hipertensión arterial y la diabetes son las Enfermedades Crónicas (EC) con mayor prevalencia en la mortalidad e incapacidad física en los individuos en etapa productiva ${ }^{(1,2)}$. Estos problemas de salud son considerados como la epidemia del siglo XXI y su principal causa de indecencia es atribuida a la alimentación poco saludable, la inactividad física, la presencia de sobrepeso y obesidad, así como al consumo de alcohol y tabaco en la población, esto cómo producto del proceso de globalización que se está viviendo actualmente ${ }^{(1-3)}$.

Instituciones gubernamentales y no gubernamentales, han mostrado una preocupación ante las EC, ya que repercuten de manera individual en ámbitos físico, mental y social; pero también impacta en los sistemas de salud, económicos y políticos de un país. Se ha hecho relevante el desarrollo de Investigación en Salud (IS) orientada a este grupo de problemas prioritarios en la población y se tienen que desarrollar intervenciones efectivas que contribuyan a prevenir y controlar las EC, para asegurar el bienestar en las personas que la padecen ${ }^{(4)}$. Las Intervenciones en Salud son complejas, ya que van dirigidas a modificar comportamientos, tienen que ser diseñadas y evaluadas, utilizando una metodología (Ensayo Clínico Aleatorizado) que permita comprobar el efecto en las personas que reciben dicha intervención ${ }^{(5)}$.

El profesional de enfermería se centra en proporcionar Intervenciones de Enfermería (IE) efectivas para producir bienestar en la parte receptora ${ }^{(6)}$. Las intervenciones constituyen un componente esencial en la disciplina de enfermería, esto permite caracterizar y distinguir la profesión de enfermería de otras disciplinas del mismo campo (Medicina, Psicología, Nutrición, etc.). Se entiende por IE, aquellas acciones (basadas en la teoría y la mejor evidencia), realizadas por el profesional de enfermería, en respuesta a problemas de salud que experimentan las personas, con el propósito de lograr una calidad de vida o bienestar del ser humano. Estás acciones se pueden llevar de manera independiente o en colaboración con el equipo de profesionales de la salud y pueden estar dirigidas a la persona, la familia y/o comunidad $(7,8)$

El enfoque más comúnmente utilizado para probar la efectividad de las intervenciones es el Ensayo Clínico Aleatorizado. Para este tipo de estudios se tiene que llevar a cabo un largo proceso que puede llevar más de 17 años, durante este tiempo se desarrollan y ejecutan diversos Estudios Pilotos para asegurar, la viabilidad, factibilidad y efectividad de un tratamiento o intervención ${ }^{(8,9)}$.

La profesión de enfermería se encuentra en proceso de transición y tiene que demostrar ser una disciplina independiente con conocimiento propio y sólido, basado en un marco metodológico riguroso y para ello tiene que comprobar que sus acciones en la práctica son efectivas y seguras. El Investigador en Enfermería debe desarrollar IE, que permita desarrollar conocimiento en el nivel de predicción (generación de tratamientos que contribuyan a mejorar las condiciones de salud de la población), estás deben de estar sustentadas en un diseño metodológico robusto, el cual permita asegurar un mejor entendimiento del fenómeno de interés de la disciplina.

\section{c. Dr. Juan Alberto López-González}

Estudiante de Doctorado en Ciencias de Enfermería. Facultad de Enfermería, Universidad de Guanajuato Maestro de Tiempo Completo. Departamento de Enfermería, Universidad de Sonora.

E-mail: juan.lopez@unison.mx

ORCID: https://orcid.org/0000-0001-5751-6137

\section{REFERENCIAS BIBLIOGRÁFICAS}

\footnotetext{
1. María J, Cortes M, Aragonés N, Godoy P, José M, Moros S. Las enfermedades crónicas como prioridad de la vigilancia de la salud pública en España. Gac Sanit. 2016;30(2):154-7.

2. The Institute for Health Metrics and Evaluation. The Institute for Health Metrics and Evaluation (IHME) [Internet]. The Institute for Health Metrics and Evaluation. 2018. Disponible en: https://vizhub.healthdata. org/gbd-compare/

3. Organización Panamerica de la Salud. Estrategia para la prevención y el control de las Enfermedades No Transmisibles [Internet]. 28.a CONFERENCIA SANITARIA PANAMERICANA 64.a SESIÓN DEL COMITÉ REGIONAL. 2012. Disponible en: http://apps.who.int/gb/archive/pdf_files/wha53/sa14.pdf

4. Instituto Nacional de Salud Pública. Prioridades de Investigación en Salud [Internet]. México: Instituto Nacional de Salud Pública; 2017.64 p. Disponible en: https://www.insp.mx/produccion-editorial/ novedades-editoriales/4512-prioridades-investigacion-salud.html

5. Craig P, Dieppe P, Macintyre S, Michie S, Nazareth I, Petticrew M. Developing and Evaluating Complex Interventions: New Guidance. Sci York [Internet]. 2008;337(a1655):a1655. Disponible en: http://discovery. ucl.ac.uk/103060/

6. Wight D, Wimbush E, Jepson R, Doi L. Six steps in quality intervention development (6SQuID). J Epidemiol Community Health. 2015;70(5):520-5

7. Sidani S, Braden CJ. Design, Evaluation, and Translation of Nursing Interventions. lowa: Wiley- BlackWell; 2011. 304 p.

8. Whittemore R, Grey M. The systematic development of nursing interventions. J Nurs Scholarsh. 2004;34(2):115-20.

9. Gitlin LN, Czaja SJ. Behavioral intervention research: Designing, evaluating, and implementing. Behavioral intervention research: Designing, evaluating, and implementing. 2016.
} 